\title{
Comparison of the clinical results of isolated Bankart and SLAP 5 lesions after arthroscopic repair
}

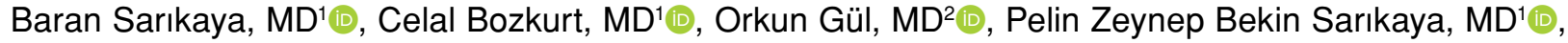 \\ Serkan Sipahioğlu, MD' ${ }^{1}$, Mehmet Akif Altay, MD'1D \\ ${ }^{1}$ Department of Orthopedics and Traumatology, Harran University Faculty of Medicine Şanlıurfa, Turkey \\ ${ }^{2}$ Department of Orthopedics and Traumatology, Karadeniz Technical University, Faculty of Medicine, Trabzon, Turkey
}

The glenohumeral joint is the most mobile joint in the human body. Shoulder stability is provided by static and dynamic stabilizers and load resistance is ensured by these stabilizers. ${ }^{[1]}$ Damage to these structures leads to dislocation and recurrent instability. Traumatic anterior instability or dislocation of the shoulder joint usually results in avulsion of the anterior inferior labrum. Bankart defined this lesion as the "essential lesion" of shoulder instability. ${ }^{[2]}$ In addition to Bankart lesions, lesions in different areas of the labrum can occur, such as superior labrum anterior to posterior (SLAP) lesions. Superior labral tears were first defined by Andrews et al. in 1985. ${ }^{[2]}$ Then in 1990, Snyder ${ }^{[3]}$ coined the term SLAP lesions.

With SLAP lesions, the superior labrum detaches along with the biceps tendon and the labral tear extends anteriorly from the posterior superior labrum. Snyder further classified SLAP lesions into four categories and over time different types of SLAP lesions were added to Snyder's classification. ${ }^{[3-5]}$ The etiology of SLAP lesions remains uncertain. There

Received: March 17, 2020

Accepted: May 18, 2020

Published online: June 18, 2020

Correspondence: Baran Sarıkaya, MD. Harran Üniversitesi Tıp Fakültesi Ortopedi ve Travmatoloji Anabilim Dalı, 63300 Şanlıurfa, Türkiye.

E-mail: baransarikaya@yahoo.com

Doi: $10.5606 /$ ehc. 2020.74750

Citation: Sarıkaya B, Bozkurt C, Gül O, Bekin Sarıkaya PZ, Sipahioğlu $S$, Altay MA. Comparison of the clinical results of isolated Bankart and SLAP 5 lesions after arthroscopic repair. Jt Dis Relat Surg 2020;31(2):223-229.

\section{ABSTRACT}

Objectives: This study aims to compare the clinical results of patients with traumatic isolated Bankart lesions and type V superior labrum anterior to posterior (SLAP) lesions after arthroscopic repair.

Patients and methods: Patients who underwent arthroscopic repair for traumatic anterior glenohumeral instability were evaluated retrospectively between December 2014 and January 2019. Fifty-one patients ( 49 males, 2 females; mean age 25 years; range, 18 to 36 years) without bone defects affecting $>20 \%$ of the glenoid fossa, off-track engaging Hills-Sachs lesions, multidirectional instability, or ligamentous laxity were included in the study. Group 1 had 31 patients with isolated Bankart lesions and group 2 had 20 patients with type V SLAP lesions. There were only two female patients in group 1 and all patients were male in group 2 . The mean age was 25 years (range, 18 to 36 years) in group 1 and 25 years (range, 19 to 35 years) in group 2. Rowe, Constant, and Western Ontario Shoulder Instability (WOSI) scoring systems were used to evaluate the clinical outcomes of the patients preoperatively and at the last follow-up.

Results: The mean follow-up time was 32 months (range, 12 to 48 months) in group 1 and 28.5 months (range, 12 to 42 months) in group 2 . There were no statistically significant differences between the two groups in terms of the number of shoulder dislocations before the surgery, mean age at the time of surgery, and the mean time from the first dislocation to surgical treatment. When the Rowe, Constant, and WOSI scores were evaluated preoperatively and at the last follow-up, there were statistically significant changes within, but not between, the two groups.

Conclusion: In type V SLAP lesions, the affected and repaired labrum surface area is larger than isolated Bankart lesions. However, as a result of appropriate surgical treatment, the affected surface area does not have a negative effect on clinical outcomes, and similar clinical results can be obtained in patients with type V SLAP lesions compared to patients with isolated Bankart lesions.

Keywords: Bankart lesion, instability, shoulder, superior labrum anterior to posterior lesion. 
are many mechanisms that can cause SLAP lesions, including a fall on an outstretched arm, instability, overhead work, and traction/tension on the biceps tendon. ${ }^{[4,6,7]}$ Previous studies have shown that the superior aspect of the labrum and the biceps anchor contribute to shoulder stability. ${ }^{[8-10]}$ Superior labrum anterior to posterior lesions may be isolated or may accompany anterior instability. Maffet et al. ${ }^{[4]}$ described the type V SLAP lesion as an anteriorinferior Bankart lesion that continues superiorly to include separation of the biceps tendon. In other words, the combination of type II SLAP lesion and Bankart lesion is defined as a type V SLAP lesion. Chronic recurrent shoulder dislocations and severe trauma are risk factors for type V SLAP lesions. ${ }^{[11]}$ In the current study, we hypothesized that type V SLAP lesions, which also affect the biceps tendon-labrum complex, would have poorer clinical outcomes than isolated Bankart lesions. Therefore, in this study, we aimed to compare the clinical results of patients with traumatic isolated Bankart lesions and type V SLAP lesions after arthroscopic repair.

\section{PATIENTS AND METHODS}

We retrospectively evaluated patients who underwent arthroscopic anatomic labrum repair for isolated Bankart and type V SLAP lesions at the Harran University Faculty of Medicine between December 2014 and January 2019. We excluded patients with accompanying "off-track" engaging Hills-Sachs lesions $(\mathrm{n}=3)$, Bankart lesions with bone defects on $>20 \%$ of the glenoid fossa $(n=1)$, multidirectional instability $(\mathrm{n}=1)$, posterior instability $(\mathrm{n}=1)$, anterior labroligamentous periosteal sleeve avulsion lesions $(n=9)$, humeral avulsion of the glenohumeral ligament lesions $(n=2)$, hyperlaxity, or rotator cuff pathologies $(\mathrm{n}=2)$. No patients were lost to follow-up. A total of 51 patients ( 49 males, 2 females; mean age 25 years; range, 18 to 36 years) were included in the study, 31 with isolated Bankart lesions (group 1) and 20 with type V SLAP lesions (group 2). All patients were male in group 2 and two were female in group 1. The mean age was 25 years (range, 18 to 36 years) in group 1 and 25 years (range, 19 to 35 years) in group 2 . The study protocol was approved by the Harran University Faculty of Medicine Ethics Committee (22/02/2019-E.8515). A written informed consent was obtained from each patient. The study was conducted in accordance with the principles of the Declaration of Helsinki.

All patients were asked questions about the mechanism of trauma, the number of dislocations, where and how reduction was performed, and how old they were when the first dislocation occurred. The patients then underwent a detailed physical examination. Joint range of motion (ROM) was measured, and anterior relocation, apprehension, and $\mathrm{O}^{\prime}$ Brien tests were performed on all patients preand postoperatively. Anteroposterior, scapula Y, and axial shoulder radiographs were routinely performed. The presence of the isolated Bankart or type $\mathrm{V}$ SLAP lesions was confirmed via magnetic resonance imaging (MRI).

Patients were evaluated clinically using shoulder scoring systems during their routine follow-up. The Rowe, Constant, and Western Ontario Shoulder Instability (WOSI) scoring systems were used to evaluate the patients preoperatively and at the last follow-up. ${ }^{[12-14]}$ In the WOSI scoring system, the questions are divided into four sections (domains). There are 10 questions addressing physical symptoms and pain (PSS); four for sports, recreation, and work (SRWS); four for lifestyle and social functioning (LS); and three for emotional well-being (ES). The PSS, SRWS, LS, and ES were evaluated individually within the WOSI scoring system. We calculated the total WOSI score and the sub-scores of the WOSI separately. The scores are presented as percentage of a normal healthy shoulder $(0 \%$ represents no deficit and $100 \%$ the worst deficit) ${ }^{[15]}$ The results of the Rowe score were categorized as follows: excellent, 90-100; good, 75-89; fair, 51-74; or poor, $\leq 50$. The results of the Constant score were categorized as follows: excellent, 90-100; good, 80-89; fair, 70-79; or poor, $\leq 69$.

Shoulder arthroscopy was performed with the patients under general anesthesia. Patients were placed in the lateral decubitus position with the affected arm at $45^{\circ}$ of abduction and $15^{\circ}$ of flexion under longitudinal traction. The scope was inserted through the posterior portal. First, glenohumeral diagnostic arthroscopy was applied to all patients. Anterior working portals were determined with a spinal needle with an outside-in technique at the rotator interval. Then the labral defect was identified. Degenerative soft tissues were debrided and the anterior edge of the glenoid was debrided with a shaver to gain a bleeding surface for attachment of the labrum. The labroligamentous complex was released with an elevator and straight and curved rasps. The releasing procedure was performed toward the six o'clock position of the glenoid. In the presence of a type V SLAP lesion, the superior chondral surface of the glenoid was debrided until subchondral bone was seen. Stabilization of the unstable SLAP lesion was repaired with a double-load suture anchor (LUPINE Loop DS Anchor System, Depuy Mitek, MA, USA), and 
the bowstring effect of the labrum contributed to the maintenance of tension and anatomical reduction of the anteroinferior labrum. One double-loaded suture anchor was inserted in the subchondral bone of the supraglenoid tubercle, and the anchor's threads were transferred using the shuttle relay method and fixated at the posterior and anterior regions of the biceps tendon. For anteroinferior labrum repair, a doubleloaded suture anchor was inserted just medial to the glenoid edge at the five o'clock position (seven o'clock position for left shoulders) after the glenoid rim was perforated at a $45^{\circ}$ medial angle. Anchor threads were transferred and passed through the labroligamentous complex with a suture passer and both sutures were tied through the anterior portal using sliding knots. Additional anchors were inserted at the three and one o'clock positions (nine and 11 o'clock positions for left shoulders) according to the size of the labral lesion. In most patients, two anchors were required to repair the Bankart lesion (26 patients in group 1 and 14 patients in group 2). One suture anchor was sufficient for the repair of SLAP lesions.

In the postoperative period, a Velpeau bandage was applied to all patients for four weeks. On the first postoperative day, pendulum exercises and elbow flexion-extension exercises were started. In the second week, passive shoulder joint exercises were started, and active assisted joint exercises were started at three weeks postoperatively. Active shoulder exercises were started at the fourth week, followed by strengthening exercises of the shoulder girdle at the sixth week. After three months, the patients were permitted to practice noncontact sports. Full return to all sporting activities was allowed after six months, according to each individual's functional recovery.

\section{Statistical analysis}

The normality of distribution of the continuous variables was tested using the Shapiro-Wilk test. For non-normally distributed data, the Mann-Whitney $U$ test was used for the comparison of two independent groups and the Wilcoxon test was performed for the comparison of two dependent measurements. Relationships between categorical variables were investigated using the chi-square test. All statistical analyses were performed with IBM SPSS for Windows version 24.0 software (IBM Corp., Armonk, NY, USA) and a $p$ value $<0.05$ was considered to indicate statistical significance.

\section{RESULTS}

There were no statistically significant differences in terms of age between the two groups. The mean follow-up time was 32 months (range, 12 to 48 months)

\begin{tabular}{|c|c|c|c|c|c|}
\hline \multirow[b]{3}{*}{ Variable } & \multicolumn{4}{|c|}{$\begin{array}{l}\text { ABLE I } \\
\text { graphic characteristics }\end{array}$} & \multirow[b]{3}{*}{$p$} \\
\hline & \multicolumn{2}{|c|}{ Bankart $(n=31)$} & \multicolumn{2}{|c|}{ Type V SLAP (n=20) } & \\
\hline & $\mathrm{n}$ & Min-Max & $\mathrm{n}$ & Min-Max & \\
\hline Age (year) & 25 & $18-36$ & 25 & $19-35$ & 0.580 \\
\hline \multicolumn{6}{|l|}{ Gender } \\
\hline Male & 29 & & 20 & & 0.445 \\
\hline Follow-up (month) & 32 & $12-48$ & 28.5 & $12-42$ & 0.185 \\
\hline Age at the first dislocation (year) & 20 & $15-35$ & 21.5 & $15-34$ & 0.536 \\
\hline Number of dislocation & 12 & $2-60$ & 7 & $2-60$ & 0.277 \\
\hline Time to surgery (month) & 36 & $3-120$ & 24 & $3-120$ & 0.884 \\
\hline \multicolumn{6}{|l|}{ Range of motion deficit } \\
\hline External rotation & $0^{\circ}$ & $0^{\circ}-20^{\circ}$ & & $0^{\circ}-20^{\circ}$ & 0.428 \\
\hline Abduction & $0^{\circ}$ & $0^{\circ}-10^{\circ}$ & & $0^{\circ}-10^{\circ}$ & 0.419 \\
\hline Forward flexion & $0^{\circ}$ & $0^{\circ}-15^{\circ}$ & & $0^{\circ}-15^{\circ}$ & 0.273 \\
\hline \multicolumn{6}{|l|}{ Trauma mechanism } \\
\hline Epileptic seizure & 1 & & 1 & & 1.000 \\
\hline Contact sports & 4 & & 3 & & 0.833 \\
\hline Recreational sports & 20 & & 12 & & 0.432 \\
\hline $\begin{array}{l}\text { Other traumas (car accident, } \\
\text { fall on an outstretched arm, assault) }\end{array}$ & 6 & & 4 & & 0.955 \\
\hline
\end{tabular}


in group 1 and 28.5 months (range, 12 to 42 months) in group 2 . The patients' demographic characteristics are shown in Table I. There were no statistically significant differences in terms of follow-up time between or in the number of shoulder dislocations before the surgery, mean age at the time of surgery, and the mean time from the first dislocation to surgical treatment (Table I).

In terms of Rowe score, in group 1, excellent results were obtained in 25 patients, good results in three patients, and poor results in three patients. In group 2, excellent results were obtained in 16 patients, good results in three patients, and poor results in one patient. In terms of Constant score, in group 1, excellent results were obtained in 22 patients, good results in six patients, a fair result in one patient, and poor results in two patients. In group 2, excellent results were obtained in 18 patients, a good result in one patient, and a fair result in one patient. In group 1, the mean WOSI score was $30.4 \%$ preoperatively and $3.66 \%$ at the last follow-up. In group 2 the mean WOSI score was $30.1 \%$ preoperatively and $4.55 \%$ at the last follow-up. Redislocation occurred in three patients in group 1 and one patient in group 2. The ages of the patients in group 1 were 22, 24, and 27 and the age of the patient in group 2 was 25 . One patient did not want to have revision surgery. However, the other three patients chose to undergo arthroscopic revision surgery (Table II).

When the Rowe, Constant, and WOSI clinical scoring systems were evaluated, no statistically significant differences were found between groups. However, there were significant differences within each group between all three preoperative and final follow-up scores. The same was true for the PSS, SRWS, LS, and ES (Table II).

All patients were examined preoperatively and at the final follow-up in terms of shoulder ROM. External rotation was measured when the shoulder was placed in the neutral position and at $90^{\circ}$ abduction in all patients. In group 1, three patients had an external

\begin{tabular}{|c|c|c|c|c|c|}
\hline \multirow[b]{3}{*}{ Shoulder scores } & \multicolumn{2}{|r|}{$\begin{array}{l}\text { TABLE II } \\
\text { patients w }\end{array}$} & erwent & copic repai & \multirow[b]{3}{*}{$p$} \\
\hline & \multicolumn{2}{|c|}{ Bankart $(\mathrm{n}=31)$} & \multicolumn{2}{|c|}{ Type V SLAP $(n=20)$} & \\
\hline & $\%$ & Min-Max & $\%$ & Min-Max & \\
\hline Rowe 1 & 30 & $15-40$ & 30 & $15-50$ & 0.567 \\
\hline Rowe 2 & 95 & $40-100$ & 95 & $55-100$ & 0.383 \\
\hline$P$ value (within groups) & \multicolumn{4}{|c|}{$0.001^{*}$} & \\
\hline Constant 1 & 68 & $57-87$ & 71.5 & $56-82$ & 0.075 \\
\hline Constant 2 & 94 & $77-100$ & 94 & $88-100$ & 0.566 \\
\hline$P$ value (within groups) & \multicolumn{4}{|c|}{$0.001^{*}$} & \\
\hline WOSI 1 (\%) & 30.4 & $10-59.1$ & 30.1 & $16.4-72.6$ & 0.736 \\
\hline WOSI 2 (\%) & 3.66 & $0.47-22$ & 4.55 & $1.09-32.7$ & 0.284 \\
\hline$P$ value (within groups) & \multicolumn{4}{|c|}{$0.001^{*}$} & \\
\hline PSS1 (\%) & 12.5 & $4.9-36.1$ & 12.5 & $4.9-44.2$ & 0.985 \\
\hline PSS2 (\%) & 1.7 & $0-12.5$ & 1.75 & $0.1-15.5$ & 0.589 \\
\hline$P$ value (within groups) & \multicolumn{4}{|c|}{$0.001^{*}$} & \\
\hline SRWS1 (\%) & 54 & $12.5-100$ & 50.6 & $30.5-100$ & 0.854 \\
\hline SRWS2 (\%) & 5.75 & 0-32 & 6.88 & $1.75-44.2$ & 0.259 \\
\hline$P$ value (within groups) & \multicolumn{4}{|c|}{$0.001^{*}$} & \\
\hline LS1 (\%) & 23.5 & $4.25-70$ & 25.7 & $6.5-75.7$ & 0.493 \\
\hline LS2 (\%) & 2.75 & $0-21.5$ & 2.88 & $1-20.5$ & 0.969 \\
\hline$P$ value (within groups) & \multicolumn{4}{|c|}{$0.001^{*}$} & \\
\hline ES1 (\%) & 67 & 24.3-99 & 62.65 & $26.6-100$ & 0.938 \\
\hline ES2 (\%) & 9 & $0-52$ & 7 & $1.33-87.6$ & 0.493 \\
\hline$P$ value (within groups) & \multicolumn{4}{|c|}{$0.001^{*}$} & \\
\hline
\end{tabular}


rotation limitation and three patients had a forward flexion limitation not exceeding $10^{\circ}$ (two of them were the same patients). In group 2, four patients had an external rotation limitation not exceeding $15^{\circ}$ and three of these four patients had a limitation in forward flexion not exceeding $10^{\circ}$. There were no statistically significant differences between the two groups in terms of external rotation $(\mathrm{p}=0.428)$ and forward flexion limitations $(p=0.884)$. No ROM limitation was detected in any patient beyond a 14-month follow-up. At the last follow-up, abduction and internal rotation of the affected shoulder were the same as they were preoperatively for all patients.

None of the patients had any complications in the early postoperative period. Redislocation occurred in four out of 51 patients. Three of these patients were in group 1 and one was in group 2. Redislocation occurred because one of the patients in group 1 had an epileptic seizure. The other three patients had a history of sports trauma. When the groups were evaluated in terms of trauma mechanism, no statistically significant difference was found between the two groups $(\mathrm{p}=0.765)$ (Table I). In four patients, traumatic redislocations occurred between 12 and 16 months following the initial surgery. A patient with epilepsy did not want to have surgery again. The other three patients opted to undergo arthroscopic revision surgery. Redislocation was not observed in the follow-up of these patients. At the last follow-up, good results were obtained in terms of Rowe and Constant scores in all three patients, the total WOSI scores were between 3.66 to $7.95 \%$, and the patients had external rotation and forward flexion limitations not exceeding $10^{\circ}$.

\section{DISCUSSION}

Maffet et al. ${ }^{[4]}$ first described the type V SLAP lesion, which is the coexistence of a type II SLAP and Bankart lesion. They also reported a rate of co-incidence of SLAP/Bankart lesions of $17 \%$ in their case series. This rate is about 11 to $27 \%$ in the literature. ${ }^{[2,6,16-}$ ${ }^{18]}$ Type V SLAP lesions were detected in $36.6 \%$ of cases in Durban et al., ${ }^{[19]} 40 \%$ in Gül et al., ${ }^{[17]}$ and $34 \%$ in Aydin et al. ${ }^{[20]}$ studies. In our case series, it was approximately $39 \%$, which is in accordance with the literature.

Type V SLAP lesions affect more labral area than Bankart lesions and may occur as a result of untreated Bankart lesions. Some studies have suggested that a longer interval between injury and surgery and the number of dislocations play a significant role in the size of the lesion and the development of a type V SLAP lesion. ${ }^{[11,18]}$ Our results do not support the results of these studies. We did not detect significant differences in the number of dislocations and the time to surgery between the two groups. The amount of affected labral surface area may be more related to the mechanism or severity of the trauma. Other studies have reported that the affected labral area is not correlated with the progression of the labral injury caused by the initial trauma but is rather correlated with the severity of the initial trauma. ${ }^{[1,21]}$ In our study, there were no significant differences between the two groups in terms of trauma mechanism. Although the mechanisms of trauma causing the labral injuries were similar, the severity of the trauma could be different between the two groups.

Although the area of labral repair was greater in patients with type V SLAP lesions, we did not find any significant differences in ROM at the last follow-up. We also found that the number of dislocations did not affect ROM when the groups were compared. A few similar studies in the literature have shown that ROM deficits are not associated with the presence of a SLAP lesion and the number of dislocations. ${ }^{[6,11,18]} \mathrm{A}$ similar study by Cho et al. ${ }^{[16]}$ that evaluated ROM between the sixth and ninth postoperative week found that ROM was remarkably low in patients with type V SLAP lesions compared to isolated Bankart lesions. They stated that the recovery of normal ROM was slower in patients with type V SLAP lesions, but that there were no significant differences between the groups in the ROM measured at the last follow-up. In our study, there were no significant differences in ROM between the groups at the last follow-up at 12-14 months. We did not detect any ROM limitations in patients with a follow-up of more than 14 months. When Kim et al. ${ }^{[21]}$ compared three groups that had Bankart lesions, Bankart and SLAP lesions, and circumferential-labral lesions, they found no significant differences in ROM. They suggested that surgeons should be careful to not cause excessive tension injury during multiple anchor fixations in extensive labral lesions.

In our study, we avoided closure at the rotator interval, increased the tension during the anterosuperior labrum repair, and considered a full release of the capsulolabral complex from the anterior glenoid neck to better mobilize the capsule. ${ }^{[2]}$ Furthermore, we did not use a trans-rotator cuff portal, which can cause postoperative stiffness after SLAP lesion repair. ${ }^{[22,23]}$

To qualitatively evaluate the clinical results in the two groups in our study, we used the Rowe, Constant, and WOSI scoring systems. A limited number of studies have compared the clinical results of isolated Bankart and type V SLAP lesions, and a 
variety of shoulder scoring systems have been used to evaluate clinical outcomes. Aydin et al. ${ }^{[20]}$ used the Constant and Rowe scoring systems, Durban et al. ${ }^{[19]}$ used the Rowe scoring system, Cho et al. ${ }^{[16]}$ used the American Shoulder and Elbow Surgeon and Rowe scoring systems, and Hantes et al. ${ }^{[18]}$ used the Constant and Rowe scoring systems. None of these studies found a significant difference between the two groups in terms of clinical scoring systems. Similarly, we did not find any significant differences in the Constant and Rowe scores between the two groups. However, unlike these studies, we also compared WOSI scores in both patient groups. ${ }^{[22]}$ There are four different scoring systems in the WOSI system, including the PSS, the SRWS, the LS, and the ES. First, we calculated and compared the total WOSI score, followed by a comparison of the four subscores. We did not see any difference between the two groups in any score.

There is no clear answer as to whether the SLAP or Bankart lesion should be repaired first in type V SLAP lesions. In one study, it was preferred to perform SLAP repair first. ${ }^{[16]}$ The authors suggested that the bowstring effect of the labrum contributes to the maintenance of tension and anatomical reduction of the anteroinferior labrum. Aydin et al. ${ }^{[20]}$ advocated that repair of the anteroinferior labral lesion should occur first to perform the best reduction of the labral tears. Some studies have claimed that if the SLAP repair is performed first, it can be difficult to clearly visualize the anterior labrum, and that the "pseudolaxity" provided by the SLAP lesion improves the visibility and working space during anterior labral repair. ${ }^{[24,25]}$ In our study, we preferred to perform the SLAP repair before the Bankart repair in the case of type V SLAP lesions. In the case of unstable SLAP lesions associated with the Bankart lesions, we believe that primary SLAP repair facilitates the reduction of the anteriorinferior labrum to the glenoid edge.

The reported recurrence rate after anterior instability surgery varies in the literature. Recent studies have reported recurrence rates of 4 to $19 \%$ after arthroscopic Bankart repair. ${ }^{[26,27]}$ In similar studies that have compared isolated Bankart and accompanying SLAP lesions, recurrence rates of $4.2 \%$ and $8.7 \%$ have been reported. ${ }^{[19,20]}$ In our study, this rate was $7.3 \%$. There are many factors that increase the risk of recurrence after anterior instability surgery and cause unsuccessful results. Age, bony defects, participation in highly demanding sports activities, ligamentous laxity, number of suture anchors, technical errors, insufficient soft tissue tensioning, patient self-control, and postoperative rehabilitation are among the main factors. ${ }^{[28]}$ In the present study, redislocation was detected in four of 51 patients. Three of these patients were in group 1 and one was in group 2. All of the recurrences in our patients were due to trauma, including one epileptic seizure and three sports injuries. These four patients had no complaints of subluxation or instability until the trauma. Three anchors were used for anterior instability in all of these patients and an additional anchor was used for SLAP repair in group 2. Some authors have reported that three or fewer anchors used in anterior instability surgery increase the risk of revision, while others have reported no significant differences between two and four anchors in terms of revision. ${ }^{[27,28]}$ After applying the anchors, we examined the capsulolabral structure with the arthroscopic probe to evaluate stabilization and applied additional anchors if needed.

The limitations of this study included a small study population and a relatively short follow-up period. In addition, postoperative radiological and MRI imaging and an evaluation of scar tissue in the labrum should be included in future studies.

In conclusion, in type V SLAP lesions, the affected and repaired labrum surface area is larger than isolated Bankart lesions. However, as a result of appropriate surgical treatment, the affected surface area does not have a negative effect on clinical outcomes, and similar clinical results can be obtained in patients with type V SLAP lesions compared to patients with isolated Bankart lesions.

\section{Declaration of conflicting interests}

The authors declared no conflicts of interest with respect to the authorship and/or publication of this article.

\section{Funding}

The authors received no financial support for the research and/or authorship of this article.

\section{REFERENCES}

1. Abboud JA, Soslowsky LJ. Interplay of the static and dynamic restraints in glenohumeral instability. Clin Orthop Relat Res 2002;400:48-57.

2. Andrews JR, Carson WG Jr, McLeod WD. Glenoid labrum tears related to the long head of the biceps. Am J Sports Med 1985;13:337-41.

3. Snyder SJ, Karzel RP, Del Pizzo W, Ferkel RD, Friedman MJ. SLAP lesions of the shoulder. Arthroscopy 1990;6:274-9.

4. Maffet MW, Gartsman GM, Moseley B. Superior labrumbiceps tendon complex lesions of the shoulder. Am J Sports Med 1995;23:93-8.

5. Modarresi S, Motamedi D, Jude CM. Superior labral anteroposterior lesions of the shoulder: part 2, mechanisms and classification. AJR Am J Roentgenol 2011;197:604-11. 
6. Kim TK, Queale WS, Cosgarea AJ, McFarland EG. Clinical features of the different types of SLAP lesions: an analysis of one hundred and thirty-nine cases. J Bone Joint Surg [Am] 2003;85:66-71.

7. Snyder SJ, Banas MP, Karzel RP. An analysis of 140 injuries to the superior glenoid labrum. J Shoulder Elbow Surg 1995;4:243-8.

8. Pagnani MJ, Deng XH, Warren RF, Torzilli PA, Altchek DW. Effect of lesions of the superior portion of the glenoid labrum on glenohumeral translation. J Bone Joint Surg [Am] 1995;77:1003-10.

9. Rodosky MW, Harner CD, Fu FH. The role of the long head of the biceps muscle and superior glenoid labrum in anterior stability of the shoulder. Am J Sports Med 1994;22:121-30.

10. Sakurai G, Ozaki J, Tomita Y, Nishimoto K, Tamai S. Electromyographic analysis of shoulder joint function of the biceps brachii muscle during isometric contraction. Clin Orthop Relat Res 1998;354:123-31.

11. Gartsman GM, Roddey TS, Hammerman SM. Arthroscopic treatment of anterior-inferior glenohumeral instability. Two to five-year follow-up. J Bone Joint Surg [Am] 2000;82:991-1003.

12. Constant CR, Murley AH. A clinical method of functional assessment of the shoulder. Clin Orthop Relat Res 1987;214:160-4.

13. Michener LA, McClure PW, Sennett BJ. American Shoulder and Elbow Surgeons Standardized Shoulder Assessment Form, patient self-report section: reliability, validity, and responsiveness. J Shoulder Elbow Surg 2002;11:587-94.

14. Rowe CR, Patel D, Southmayd WW. The Bankart procedure: a long-term end-result study. J Bone Joint Surg [Am] 1978;60:1-16.

15. Salomonsson B, Ahlström S, Dalén N, Lillkrona U. The Western Ontario Shoulder Instability Index (WOSI): validity, reliability, and responsiveness retested with a Swedish translation. Acta Orthop 2009;80:233-8.

16. Cho HL, Lee CK, Hwang TH, Suh KT, Park JW. Arthroscopic repair of combined Bankart and SLAP lesions: operative techniques and clinical results. Clin Orthop Surg 2010;2:39-46.

17. Gül O, Okutan AE, Ayas MS. Arthroscopic glenoid labral lesion repair using all-suture anchor for traumatic anterior shoulder instability: short-term results. J Shoulder Elbow Surg 2019;28:1991-7.
18. Hantes ME, Venouziou AI, Liantsis AK, Dailiana ZH, Malizos KN. Arthroscopic repair for chronic anterior shoulder instability: a comparative study between patients with Bankart lesions and patients with combined Bankart and superior labral anterior posterior lesions. Am J Sports Med 2009;37:1093-8.

19. Durban CM, Kim JK, Kim SH, Oh JH. Anterior Shoulder Instability with Concomitant Superior Labrum from Anterior to Posterior (SLAP) Lesion Compared to Anterior Instability without SLAP Lesion. Clin Orthop Surg 2016;8:168-74.

20. AydinN,UnalMB, AsansuM,TokO.ConcomitantSLAPrepair does not influence the surgical outcome for arthroscopic Bankart repair of traumatic shoulder dislocations. J Orthop Surg (Hong Kong) 2017;25:2309499017718952.

21. Kim DS, Yi CH, Kwon KY, Oh JR. Relationship between the extent of labral lesions and the frequency of glenohumeral dislocation in shoulder instability. Knee Surg Sports Traumatol Arthrosc 2013;21:430-7.

22. Atik OŞ. Is there something new and interesting in my article? Eklem Hastalik Cerrahisi 2019;30:69.

23. Cohen DB, Coleman S, Drakos MC, Allen AA, O'Brien SJ, Altchek DW, et al. Outcomes of isolated type II SLAP lesions treated with arthroscopic fixation using a bioabsorbable tack. Arthroscopy 2006;22:136-42.

24. Lo IK, Burkhart SS. Triple labral lesions: pathology and surgical repair technique-report of seven cases. Arthroscopy 2005;21:186-93.

25. Morgan CD, Burkhart SS, Palmeri M, Gillespie M. Type II SLAP lesions: three subtypes and their relationships to superior instability and rotator cuff tears. Arthroscopy 1998;14:553-65.

26. Lee $\mathrm{SH}, \mathrm{Lim} \mathrm{KH}, \mathrm{Kim}$ JW. Risk factors for recurrence of anterior-inferior instability of the shoulder after arthroscopic bankart repair in patients younger than 30 years. Arthroscopy 2018;34:2530-6.

27. Voos JE, Livermore RW, Feeley BT, Altchek DW, Williams RJ, Warren RF, et al. Prospective evaluation of arthroscopic bankart repairs for anterior instability. Am J Sports Med 2010;38:302-7.

28. Porcellini G, Campi F, Pegreffi F, Castagna A, Paladini P. Predisposing factors for recurrent shoulder dislocation after arthroscopic treatment. J Bone Joint Surg [Am] 2009;91:2537-42. 